\title{
GABA, A Primary Metabolite Controlled by the Gac/Rsm Regulatory Pathway, Favors a Planktonic Over a Biofilm Lifestyle in Pseudomonas protegens CHAO
}

\author{
Kasumi Takeuchi ${ }^{\dagger}$ \\ Division of Plant and Microbial Sciences, Institute of Agrobiological Sciences, National Agriculture and Food Research \\ Organization, 2-1-2 Kannondai, Tsukuba, Ibaraki 305-8602, Japan
}

Accepted 4 October 2017.

\begin{abstract}
In Pseudomonas protegens CHA0 and other fluorescent pseudomonads, the Gac/Rsm signal transduction pathway is crucial for the expression of secondary metabolism and the biological control of fungi, nematodes, and insects. Based on the findings of a previous metabolomic study, the role of intracellular $\boldsymbol{\gamma}$-aminobutyrate (GABA) as a potential signal in the Gac/Rsm pathway was investigated herein. The function and regulation of a $g a b D T$ (c01870-c01880) gene cluster in strain CHA0 were described. The gabT gene encoded GABA transaminase (GABAT) and enabled the growth of the bacterium on GABA, whereas the upstream gabD gene (annotated as a gene encoding succinic semialdehyde dehydrogenase) had an unknown function. A gacA mutant exhibited low GABAT activity, leading to the markedly greater intracellular accumulation of GABA than in the wild type. In the gacA mutant, the RsmA and RsmE proteins caused translational gabD repression, with concomitant gabT repression. Due to very low GABAT activity, the gabT mutant accumulated GABA to high levels. This trait promoted a planktonic lifestyle, reduced biofilm formation, and favored root colonization without exhibiting the highly pleiotropic gacA phenotypes. These results suggest an important role of GABA in the Gac/Rsm-regulated niche adaptation of strain CHA0 to plant roots.
\end{abstract}

Pseudomonas protegens CHA0 is an effective biocontrol bacterium that has adapted to the rhizosphere, in which it suppresses plant diseases by producing several extracellular enzymes and secondary metabolites with antibiotic activity. These exoproducts contribute to plant protection by strain $\mathrm{CHA} 0$ and other root-colonizing Pseudomonas species exhibiting biocontrol activity. The expression of these biocontrol factors depends on the Gac/Rsm signal transduction pathway (Kidarsa et al. 2013; Lapouge et al. 2008). Mutants defective in the Gac/Rsm signal transduction pathway have a reduced ability to synthesize biocontrol factors, suppress plant diseases, and produce insecticides (Haas and Défago 2005; Kim et al. 2011; Kupferschmied et al. 2013). The Gac/Rsm signal transduction pathway also affects other phenotypes of multicellular behavior, such as biofilm formation and swarming motility (Kidarsa et al. 2013; Lapouge et al. 2008; Song et al. 2016). The Gac/Rsm system is involved in a switch between a planktonic and biofilm mode of growth (Valentini

\section{${ }^{\dagger}$ Corresponding author: Kasumi Takeuchi; E-mail: kasumit@affrc.go.jp}

*The $\boldsymbol{e}$-Xtra logo stands for "electronic extra" and indicates that three supplementary figures and four supplementary tables are published online.

○ 2018 The American Phytopathological Society and Filloux 2016). In the case of biocontrol strains of Pseudomonas spp., this switch enables bacteria to proliferate on plant roots and, subsequently, to compete effectively with other rhizosphere organisms during a sessile mode of growth (Barahona et al. 2011; Lalaouna et al. 2012). This switch is positively regulated by guanosine tetraphosphate (ppGpp), an intracellular signal characteristic of nutrient limitation and stress (Takeuchi et al. 2012). Furthermore, the implementation of a sessile lifestyle typically requires bis- $\left(3^{\prime}-5^{\prime}\right)$-cyclic dimeric GMP (c-di-GMP) as an important intracellular signal in pseudomonads as well as in other bacteria (Valentini and Filloux 2016).

The Gac/Rsm signal transduction pathway is initiated by the GacS/GacA two-component system (Lapouge et al. 2008; Valentini and Filloux 2016). At high cell population densities, the GacS sensor kinase is autophosphorylated; this enables activation of the cognate GacA response regulator, leading to the transcription of two or more noncoding small RNAs (sRNAs) in Pseudomonas spp. (Kay et al. 2005, 2006; Lalaouna et al. 2012). In P. protegens strains CHA0 and Pf-5 as well as in P. brassicacearum, GacA positively controls the expression of three sRNAs termed RsmX, RsmY, and RsmZ (Kay et al. 2005; Kidarsa et al. 2013; Lalaouna et al. 2012). These sRNAs exhibit high affinity for the RNAbinding protein RsmA and its paralogue RsmE (Heeb et al. 2002; Kay et al. 2005; Reimmann et al. 2005; Valverde et al. 2003). The RsmA and RsmE proteins repress the translation of genes having a conserved ANGGAN motif in or near the Shine-Dalgarno sequence; in vitro, this repression typically occurs during rapid growth (Lapouge et al. 2008; Reimmann et al. 2005). When $\mathrm{RsmX} / \mathrm{Y} / \mathrm{Z}$ sRNAs are induced during restricted growth at a high cell population density, they relieve the translational repression of target genes by sequestering the RsmA and RsmE proteins. Thus, the biofilm state and secondary metabolism are favored (Lapouge et al. 2008; Valentini and Filloux 2016). A multitude of regulatory proteins, including the antagonistic sensor RetS and agonistic sensor LadS, modulate the output of the Gac/Rsm pathway (Goodman et al. 2009; Humair et al. 2009; Valentini and Filloux 2016). In strain CHA0, evidence has been obtained for the physical interaction between RetS and GacS (Workentine et al. 2009). A direct and specific interaction between RetS and GacS has been demonstrated in Pseudomonas aeruginosa PAK, in which RetS forms heterodimers with GacS, thereby preventing the phosphorylation of GacA (Goodman et al. 2009).

In a previous metabolomic study (Takeuchi et al. 2012), we noted that gacA and retS mutations exerted opposite effects on the intracellular levels of some central metabolites including ppGpp. In a gacA mutant, ppGpp accumulates to high levels, which appears to prevent the overshooting of GacA-dependent functions by 
a feedback mechanism. Another metabolite that accumulates in a gacA mutant and is relatively depleted in a retS mutant is $\gamma$ aminobutyrate (GABA) (Takeuchi et al. 2012). This finding is of particular interest because GABA is a potential signaling molecule in the rhizosphere (Badri et al. 2013) and is known to downregulate quorum sensing in the plant pathogen Agrobacterium tumefaciens (Chevrot et al. 2006). Moreover, GABA has a negative effect on the ability of Pseudomonas syringae pv. tomato DC3000 to elicit the hypersensitive reaction in tobacco (Park et al. 2010). These findings imply that GABA plays important roles as a signal molecule in plant-bacteria interactions. In bacteria, GABA originates from polyamines, mostly from putrescine (Bartsch et al. 1990; Chou et al. 2008; Dasu et al. 2006). GABA is catabolized to succinate in two steps. GABA transaminase (GABAT) encoded by the gabT gene generates succinic semialdehyde. The gabD gene specifies an $\mathrm{NAD}(\mathrm{P})^{+}$-dependent succinic semialdehyde dehydrogenase (SSDH), which oxidizes succinic semialdehyde to succinate (Bartsch et al. 1990; Chou et al. 2008).

In the present study, the mechanisms by which the Gac/Rsm system regulates GABA accumulation in $P$. protegens $\mathrm{CHA} 0$ were investigated. GABA accumulation in the gacA mutant of strain $\mathrm{CHA} 0$ suggests i) GABA production is negatively regulated by the Gac/Rsm pathway or ii) GABA catabolism is positively regulated by the Gac/Rsm pathway. Since the Gac/Rsm pathway is known to play important roles in the positive regulation of many genes (Lapouge et al. 2008), the latter is more plausible. Therefore, the hypothesis that the expression of one or both the gabT and $g a b D$ gene is positively regulated by the Gac/Rsm pathway was examined. Furthermore, to evaluate the function of GABA in interactions with plants, the role of GABA in the switch from a planktonic and to a biofilm mode of growth was examined in vitro and during root colonization.

\section{RESULTS}

Construction of gabD and gabT mutants in strain CHAO.

In the genome of strain CHA0, the genes PFLCHA0_c01880, PFLCHA0_c48650, and PFLCHA0_c49850 are annotated as GABAT genes, termed gabT1, gabT2, and gabT3, respectively (Pseudomonas Genome database; Winsor et al. 2011). Among the products of these three genes, the homolog of GabT1 has been functionally characterized in the most detail as GABAT in Escherichia coli K-12 (Schneider et al. 2002). The deduced amino acid sequence of GABAT in E. coli $\mathrm{K}-12$ shared 74.2, 28.5, and $41.2 \%$ identities with the gabT1, gabT2, and gabT3 gene products, respectively. The gabD locus (PFLCHA0_c01870) encoding a putative SSDH is located upstream of gabT1 (Fig. 1). Furthermore, only the homolog of gabT1 in P. syringae pv. tomato DC3000, named gabT2 in that strain and sharing $91.3 \%$ amino acid identity, has the ability to restore the GABATnegative phenotype of the triple mutant gabT2/T3/T1, whereas the other two homologs cannot (Park et al. 2010). Therefore, the gabDT1 cluster in strain CHA0 was characterized and chromosomal gabD and gabT1 (hereafter called gabT) deletion mutants were generated (Fig. 1).

GABAT activity was tested with GABA as the substrate and 2oxoglutarate as the amino-accepting oxoacid in the wild type and in the mutants. Strains were grown in defined mannitol-glutamate (MG) medium amended with $5 \mathrm{mM}$ GABA to ensure the induction of GABA catabolic functions and were harvested during the late exponential phase. The gabT mutant had strongly decreased GABAT-specific activity (Table 1), demonstrating that the gabT gene encodes the major 2-oxoglutarate-dependent GABAT in $P$. protegens CHA0. The expression of gabDT genes from the upstream region of $g a b D$ in trans restored GABAT activity, indicating that this phenotype was due to the inactivation of the $g a b T$ gene (Supplementary Table S1).
SSDH activity was also measured. The wild-type strain and the $g a b D$ mutant showed similar activities under the conditions tested (Table 1), suggesting that the gabD gene either specifies a minor SSDH or is incorrectly annotated as an SSDH gene. In the gacA mutant, both enzyme activities were lower than that of the wild type (Table 1), indicating that the levels of both enzymes are under the control of the Gac/Rsm system.

In order to confirm the function of the gabT gene in GABA catabolism, the ability of the mutant to grow on a minimal medium agar plate supplemented with $20 \mathrm{mM}$ GABA as the sole carbon and nitrogen source was analyzed (Supplementary Fig. S1). GABA supported the growth of the wild type, whereas the gabT mutant did not grow, confirming that the gabT gene is necessary for GABA catabolism. The gabD mutant grew on the same plate, similarly to the wild type, which is consistent with SSDH data (Table 1). The gacA mutant did not have any detectable growth handicap, suggesting that its reduced GABAT activity was sufficient for growth. Complementation of the gabT mutant was found to restore growth. Putrescine, a precursor of GABA in $P$. aeruginosa (Yao et al. 2011), was also examined as the sole carbon and nitrogen source and the growth patterns of all strains were similar to those found on the GABA plate. Thus, putrescine catabolism also depends on the function of the gabT gene in strain CHA0.

\section{The Gac/Rsm system regulates gabDT expression at the translational level.}

The gabDTl genes of strain CHA0 may be cotranscribed and coordinately induced by putrescine and GABA, similarly to the gabDT genes of Pseudomonas aeruginosa PAO1 (Chou et al. 2008). In order to clarify how defective GacA decreases GABAT activity and, hence, increases intracellular GABA levels (Table 1), a translational gabD'- 'lacZ fusion was constructed and its expression was measured. The gacA mutant CHA89 as well as the rsmX rsmY rsmZ triple mutant CHA1144 expressed markedly lower levels of $g a b D$ '- 'lacZ throughout growth than the wild type (Fig. 2A). The retS mutant CHA1202 and the rsmA rsmE double mutant CHA1009 showed higher levels of expression than the wild type (Fig. 2A). This expression pattern is typical of posttranscriptional control by the GacA cascade in strain CHA0 (Humair et al. 2009). When GacA is absent, the repressor RsmA/E will cause translational repression of this fusion, and the ret $S$ mutation may have the opposite effect; the absence of RetS results in the overexpression of $r s m X r s m Y r s m Z$, all of which sequester the repressor RsmA/E. The removal of RsmA/E renders the ribosome-binding site of the gabDT operon accessible for the translational machinery.

The predicted Shine-Dalgarno sequence of $g a b D$ has a potential RsmA/E binding site (UAAGGAAG) (Fig. 1A), in which the central ribonucleotides (AAGGAA) may form a hexa-loop on a $\mathrm{U}-\mathrm{G}$ base pair (underlined) and allow the binding of RsmA and RsmE (Lapouge et al. 2008). No such motif was found upstream of gabT. Assuming that $g a b D$ and $g a b T$ are cotranscribed and cotranslated, two versions of a $g a b D$ - $g a b T$ ' - 'lacZ fusion were constructed; one had the original UAAGGAAG sequence, whereas the other contained a conventional Shine-Dalgarno sequence (AGGA) (Ma et al. 2002) (Fig. 1B). As shown in Figure 2B, gabD-gabT'- 'lacZ expression was significantly weaker in the gacA mutant than in the wild type (Fig. 2B), with a $25 \%$ reduction being observed at an optical density at $600 \mathrm{~nm}\left(\mathrm{OD}_{600}\right)$ of approximately 1.7 (Miller units of CHA0 and the gacA mutant were $800 \pm 18$ and $601 \pm 12$, respectively). In contrast, the construct that had a conventional $g a b D$ Shine-Dalgarno sequence essentially lost the regulation of gabD-gabT' - 'lacZ by GacA (Fig. 2B), with a $9 \%$ reduction being observed at the same growth phase (Miller units of CHA0 and the gacA mutant were $639 \pm 18$ and $579 \pm 8$, respectively). This noncanonical translational control of the downstream gabT gene 
may be attributed to translational coupling; however, this was not examined in detail in the present study. The mutation introduced into the RsmA/E binding site reduced the expression of the lac $Z$ reporter in the wild type, and a possible reason for this is that the mutant Shine-Dalgarno sequence is weaker than the wild-type sequence. Another explanation is that the wild-type leader sequence appears to be optimal for mRNA stability, which is consistent with previous findings on hcnA expression (Blumer and Haas 2000; Lapouge et al. 2007).

\section{Intracellular GABA concentrations \\ in strain CHAO and its mutants.}

Intracellular GABA levels were assessed by high-performance liquid chromatography (HPLC) (Table 1). The concentration of GABA was $94.5 \pm 4.3$ pmol per optical density at $600 \mathrm{~nm}$ $\left(\mathrm{OD}_{600 \mathrm{~nm}}\right)$ unit (one $\mathrm{OD}_{600 \mathrm{~nm}}$ unit corresponds to approximately $1.0 \times 10^{9}$ cells per milliliter) in wild-type CHA0 grown in $\mathrm{MG}+\mathrm{GABA}$ medium to the late exponential phase (at an $\mathrm{OD}_{600}$ of approximately 1.7) (Supplementary Fig. S2). In the gabT mutant, GABA accumulation was markedly greater than that in the wild type, as expected from the GABAT assays and growth tests (Table 1). The $g a b D$ and $g a c A$ mutants showed higher levels of GABA than the wild type, which is consistent with GABAT data (Table 1). The elevated GABA level observed in the $g a b D$ mutant may be due to a polar effect caused by the deletion of $g a b D$ on the expression of the downstream gabT gene. It is important to note that, in the original metabolomic study (Takeuchi et al. 2012), the gacA mutant also showed elevated GABA levels after growth in medium not amended with GABA. Although the medium used (diluted and modified glycerol-casamino acid medium) was different from that in the present study, the concentration of GABA was $34 \pm 2.5$ pmol per OD $_{600 \mathrm{~nm}}$ unit in wild-type $\mathrm{CHA} 0$ and $147 \pm 47$ pmol per $\mathrm{OD}_{600 \mathrm{~nm}}$ unit in the gacA mutant at the late exponential phase (Takeuchi et al. 2012).

In the medium used (MG + GABA), the gabT and gabD mutants grew at wild-type rates. In this experiment, the gacA mutant resumed growth without a lag phase, giving it a growth advantage over the other strains. This growth advantage in the gacA mutant was also observed in MG medium without amended GABA (data not shown).

\section{GABA affects planktonic growth, biofilm formation,} and root colonization.

It has not yet been clarified whether the accumulation of GABA affects bacterial phenotypes involved in rhizosphere colonization, specifically, the switch between the motile planktonic and sessile modes of growth. In vitro, the gacA mutant had a reduced ability to form biofilms and remained longer in a planktonic phase than

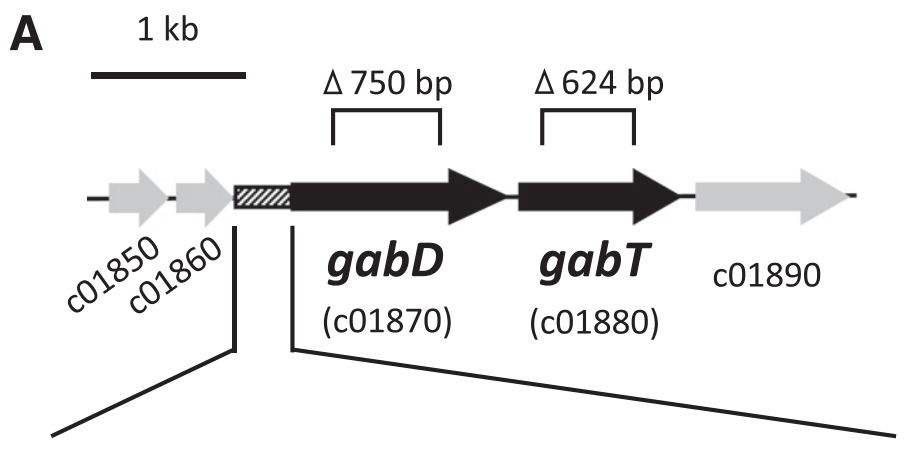

CCGTTCCCCAGGCGCCATGCCAGCGCCCCCGCCGCCTGCCATAGGCTGCGGGGGCGTTGT

CTTTTGCCCGGCGGCATGGGAGGTTGAAGCGGATTTTTTACCGCTAGGCGCCTTTACGTT CTTTTTTTCGAACAGCCTATTGTCCTTTACCCCCGTTGACCCTAGGATTCGTTTGAGATT TCAAACGCTCTTGCCTGGGTGCCCAAACGCACGTCTATTCTTGTGTGCGACTTTTTCGTG CTTTTACCCAGTGAACGATTTTCCTGACGGCAGCCTGGCCACGGGCTGGTGACAGCGCCC CGGCGCTCTCCTCCTGACCTGGCGCCTTTCTACAATCACAATTCGCCCCGCGTATGCGCG GTGCTGTTAAGGAAGCCGACATG
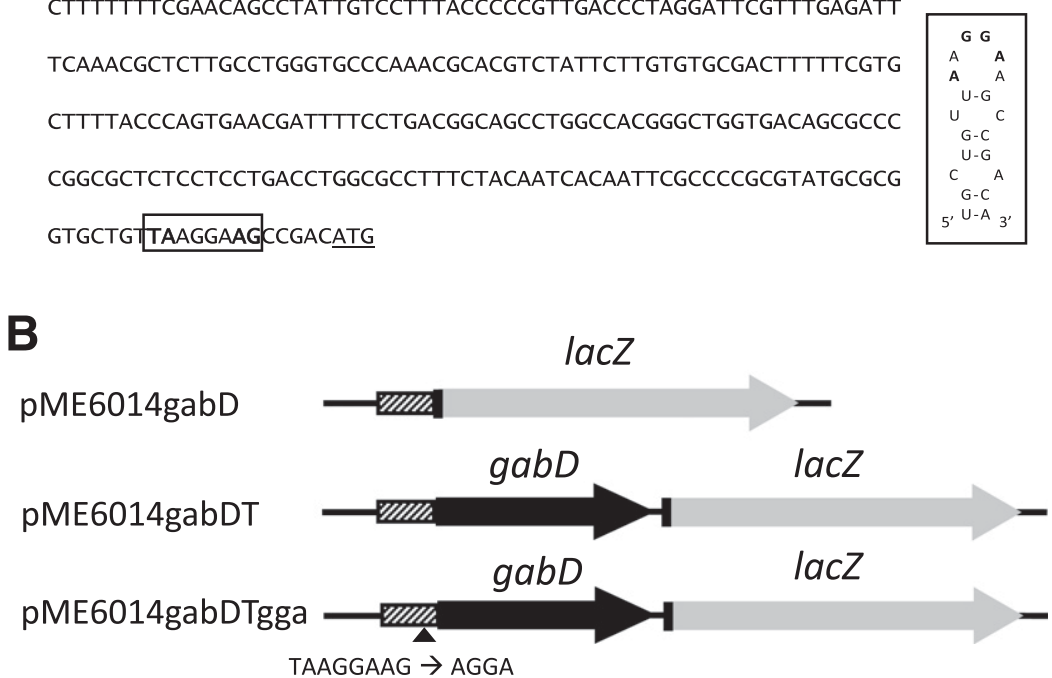

Fig. 1. Physical map and constructs involving gabD and gabT genes of Pseudomonas protegens CHA0. A, Construction of gabD and gabT mutants. $\Delta$ represents the regions deleted in $g a b D$ or gabT. The 380-bp intergenic region between PFLCHA0_c01860 and gabD is shown as a hatched box and its nucleotide sequence is indicated below. A putative RsmA/E binding site is shown in a box upstream of the predicted ATG start codon (underlined) of gabD (according to the annotation of strain Pf-5). Inset, predicted secondary structure of the gabD mRNA leader sequence near the translation start site when complexed with RsmA/E. The typical GGA motif is shown in bold face. B, Construction of gabD'- 'lacZ and gabDT'-'lacZ fusions. The plasmids pME6014gabD, pME6014gabDT, and pME6014gabDTgga each contain a translational 'lacZ fusion in the vector pME6014. The triangle shows the site at which the predicted RsmA/E binding site of pME6014gabDT was replaced by a conventional AGGA Shine-Dalgarno sequence in pME6014gabDTgga. 
the wild type (Fig. 3), which is consistent with previous findings obtained in strain Pf-5 (Kidarsa et al. 2013) and P. aeruginosa (Parkins et al. 2001). The behavior of the gabD mutant was similar to that of wild-type CHA0 (Fig. 3), except that it showed intermediate behavior between the wild type and gabT mutant in the absence of amended GABA in planktonic cell growth (Fig. 3B and $\mathrm{C}$ ). Phenotypically, the gabT mutant was intermediate between the wild type and gacA mutant in this assay (Fig. 3). Biofilm formation by the gabT mutant was not significantly weaker than that by the wild type in the absence of amended GABA at a $P<0.05$ level (Fig. 3A). Although the intracellular GABA level in each strain without amended GABA has yet to be assessed, a threshold intracellular GABA level may be required to exert effects on the planktonic to biofilm switch. The addition of GABA to the medium enhanced the coefficient of planktonic cells over the biofilm cell mass in all strains in a dose-dependent manner (Fig. 3C), supporting the function of GABA as a signal molecule.

Although the gacA mutant had lost swarming motility (Supplementary Fig. S3), which is known to depend on Gac/Rsm function in strains CHA0 and Pf-5 (Kay et al. 2005; Kidarsa et al. 2013), the gabT mutant exhibited the ability to swarm, similarly to the wild type and $g a b D$ mutant. Unlike the biocontrol-defective gacA mutant (Kay et al. 2005), the gabT mutant had conserved biocontrol ability against the oomycete Pythium ultimum on cucumber under natural soil conditions (Supplementary Table S2), suggesting that the gabT mutant had an unabated ability to produce biocontrol factors. We then performed a gnotobiotic cucumber root colonization assay (Table 2), in which the CHA0 inoculum was $10^{4}$-fold lower than that used in the biocontrol assay to avoid the saturation of colonization. The gabT mutant displayed a significantly greater capacity for the colonization of cucumber roots than the wild type; however, these differences were small (Table 2), without showing a difference in the fresh weight of host plants (data not shown). These results suggest that GABA promotes root colonization by sustaining a planktonic mode of growth following root inoculation.

\section{DISCUSSION}

Pseudomonads have two possible modes of growth, i.e., they adopt a planktonic or sessile lifestyle. During planktonic growth, cells prioritize primary metabolism for the rapid production of cell mass and motility, whereas, in the sessile (biofilm) lifestyle, cells favor the production of extracellular matrix molecules, secreted enzymes, and secondary metabolites. This dual behavior is observed in plant-beneficial Pseudomonas spp., such as P. protegens, as well

Table 1. Specific enzyme activities and intracellular $\gamma$-aminobutyrate (GABA) concentrations in Pseudomonas protegens $\mathrm{CHA} 0$ and its mutants grown in mannitol-glutamate medium with $5 \mathrm{mM} \mathrm{GABA}^{\mathrm{a}}$

\begin{tabular}{|c|c|c|c|c|}
\hline \multirow[b]{2}{*}{ Strain } & \multirow[b]{2}{*}{ Genotype } & \multicolumn{2}{|c|}{ Specific activity } & \multirow[b]{2}{*}{$\mathbf{G A B A}^{\mathbf{d}}$} \\
\hline & & $\mathbf{G A B A T}^{\mathbf{b}}$ & SSDH $^{\mathrm{c}}$ & \\
\hline HA0 & & & & \\
\hline CHA0gabD & $\triangle g a b D$ & $14.8 \pm 6.7^{*}$ & $14.2 \pm$ & $\pm 13.6 \dagger$ \\
\hline СПАО & $g a b T$ & $2.9 \pm$ & $14.9 \pm$ & $7+1$ \\
\hline СНАХУ & $g a c A:: \mathrm{Km}^{\mathrm{r}}$ & $9.4 \pm 0.4^{*}$ & $5.7 \pm 3.8 *$ & $163.6 \pm 15.2 \dagger$ \\
\hline \multicolumn{5}{|c|}{$\begin{array}{l}\text { Data are presented as averages of three replicates } \pm \text { standard deviation. } \\
\text { b GABT }=\text { GABA transaminase; SSDH }=\text { succinic semialdehyde dehy- } \\
\text { drogenase. Measured as nanomoles glutamate per minute per milligram } \\
\text { of protein. Asterisks ( } * \text { and } * * \text { ) indicate values significantly different } \\
\text { from those in CHA0 at } P<0.05 \text { and } 0.01 \text {, respectively ( } t \text {-test). } \\
\text { Measured as nanomoles NADH per minute per milligram of protein. An } \\
\text { asterisk (*) indicates a value significantly different from that in CHA0 at } \\
P<0.05 \text { ( } t \text {-test). } \\
\text { deasured as picomoles per optical density at } 600 \mathrm{~nm} \text { unit. Daggers ( } \dagger \text { and } \\
+\dagger \text { ) indicate values significantly different from those in CHA0 at } P< \\
0.005 \text { and } 0.00005 \text {, respectively ( } t \text {-test). }\end{array}$} \\
\hline
\end{tabular}

as in plant- or animal-pathogenic species such as $P$. aeruginosa (Kay et al. 2005; Kidarsa et al. 2013; Lapouge et al. 2008; Laville et al. 1992; Parkins et al. 2001; Valentini and Filloux 2016). The switch from planktonic to sessile growth is a highly complex, structured process that requires, in addition to an appropriate surface, the function of a plethora of Pseudomonas genes. In the hierarchy of master regulators that orchestrate this switch, the GacS/GacA two-component system occurs at the top (Valentini and Filloux 2016), resulting in the highly pleiotropic phenotypes of gacS/gacA mutants (Lapouge et al. 2008). Numerous extraand intracellular signals (or cues) were expected to participate in
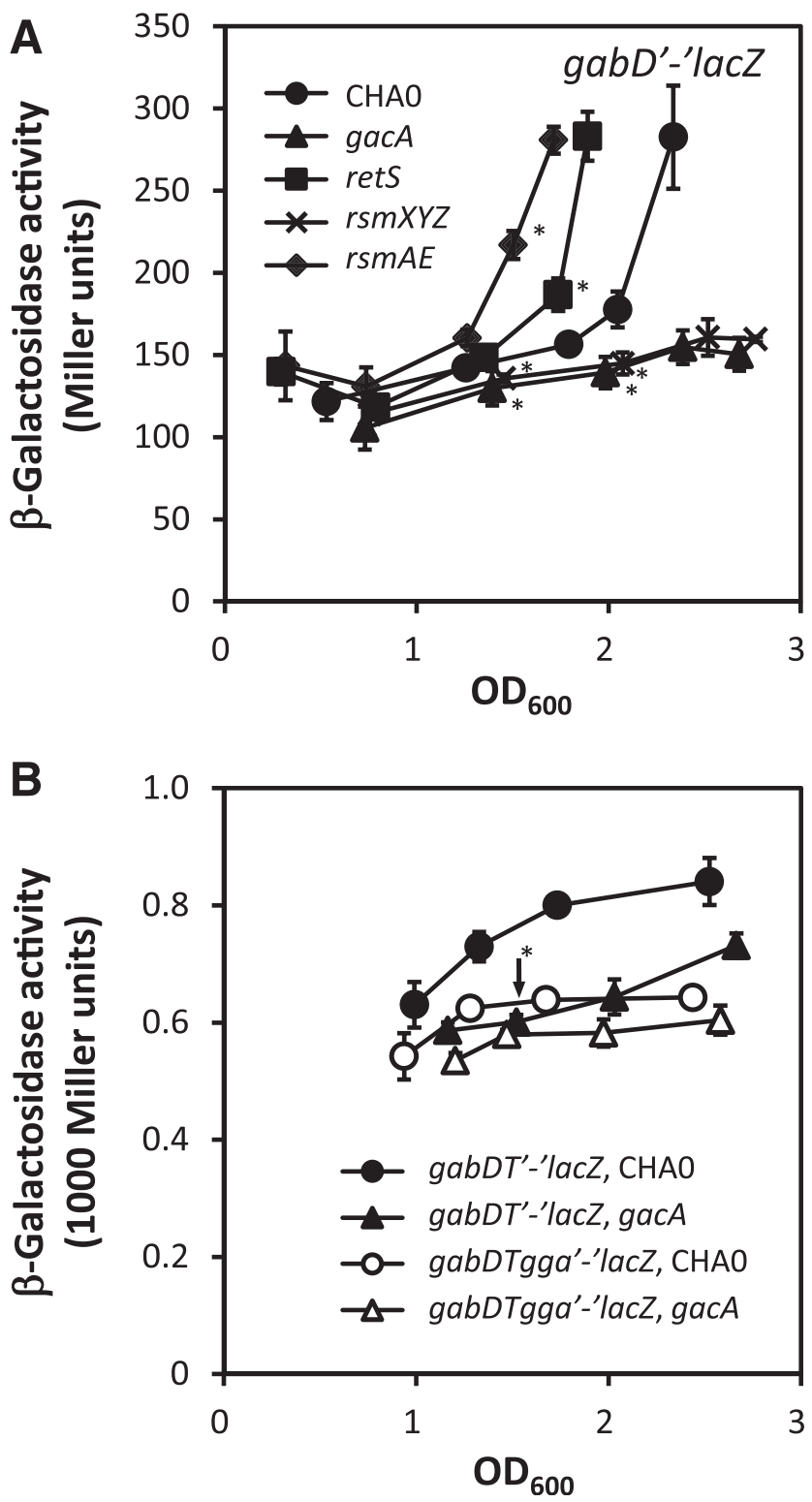

Fig. 2. Expression of gabD'- 'lacZ and gabDT'-'lacZ fusions in CHA0 and in Gac/Rsm mutants. A, Expression of gabD'-'lacZ on pME6014gabD in CHAO and in gacA, retS, rsmAE, and $\operatorname{rsmXYZ}$ mutants grown in nutrient yeast broth (NYB). The Student's $t$ test was performed to compare each mutant with $\mathrm{CHA} 0$ in the late exponential phase (at an optical density at $600 \mathrm{~nm}\left[\mathrm{OD}_{600}\right]$ of approximately 1.7$)$. Asterisks $(*)$ indicate values significantly different at $P<0.05$. B, Expression of gabD-gabT'-'lacZ on pME6014gabDT and of gabD-gabT'(gga)-'lacZ on pME6014gabDTgga in $\mathrm{CHA} 0$ and the gacA mutant grown in NYB. The symbols indicate the averages of triplicate cultures and error bars indicate standard deviations. The Student's $t$ test was performed to compare the gacA mutant with CHA0 in each construct in the late exponential phase (at an $\mathrm{OD}_{600}$ of approximately 1.7). An asterisk (*) indicates values significantly different at $P<0.05$ (with an arrow). 
the switch under GacS/GacA control. However, limited information is currently available on these low-molecular weight effectors. As described above, the intracellular starvation and stress signal ppGpp has a positive effect on the function of the Gac/Rsm cascade in P. protegens (Takeuchi et al. 2012) and there is ample evidence for intracellular c-di-GMP playing an executive role in the Gac/Rsm cascade and, hence, in the establishment of biofilms of $P$. aeruginosa (Kulasakara et al. 2006; Valentini

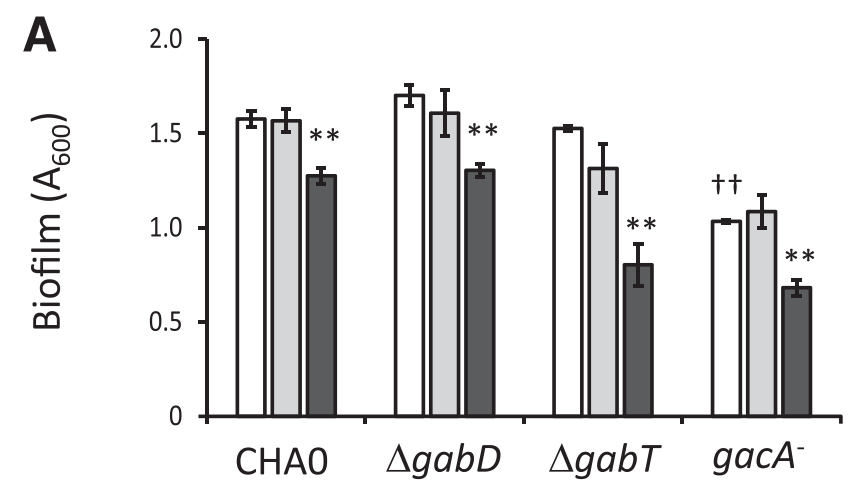

B
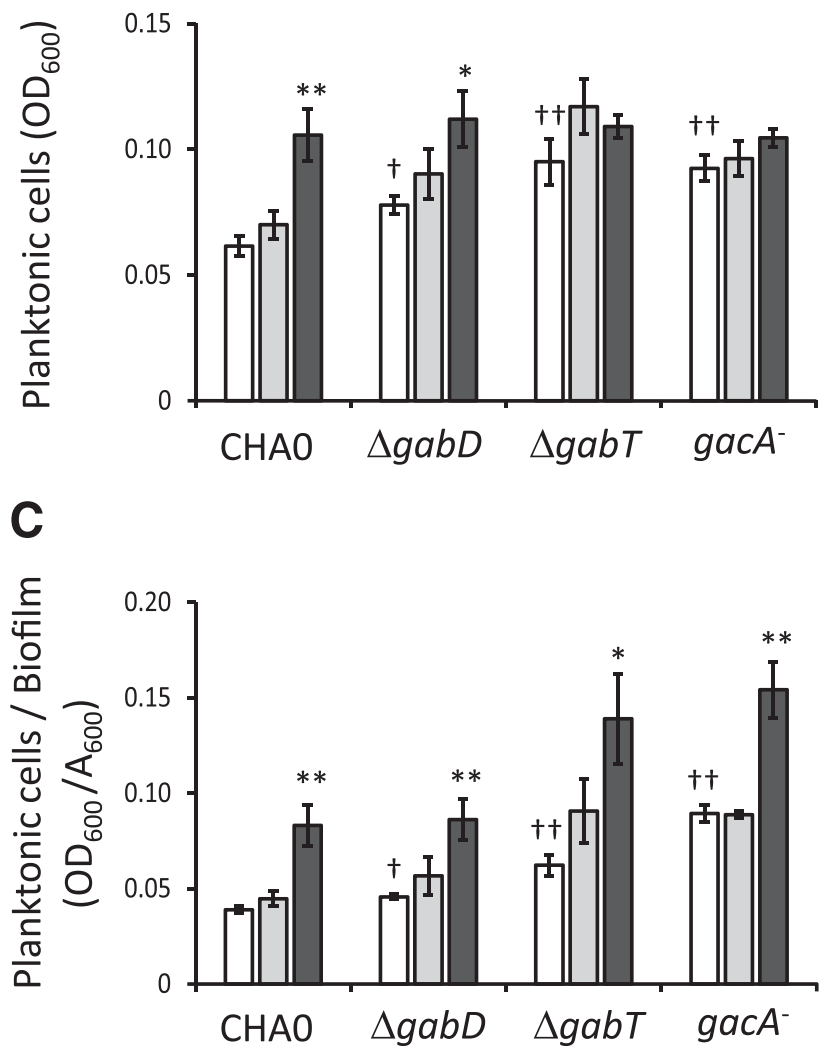

Fig. 3. Effects of $\gamma$-aminobutyrate (GABA) on the growth of planktonic cells and biofilm formation by Pseudomonas protegens $\mathrm{CHA} 0$ and its mutants. Cells were grown in mannitol-glutamate medium without added GABA (white bar), with $2 \mathrm{mM}$ GABA (light gray bar), or with $5 \mathrm{mM}$ GABA (dark gray bar). A, Biofilm formation on polypropylene, as assessed by crystal violet staining. B, Planktonic cell growth, as assessed by measuring optical density at $600 \mathrm{~nm}$ in a liquid culture. C, Ratio of planktonic cell growth to biofilm formation. The means and standard deviations from triplicate experiments are shown. The Student's $t$ test was performed to compare each strain treated with $5 \mathrm{mM}$ GABA with its respective control strain without added GABA; asterisks (* and **) indicate values significantly different at $P<0.05$ and 0.01 , respectively. The Student's $t$ test was also performed to compare each mutant with $\mathrm{CHA} 0$ at $0 \mathrm{mM}$ added GABA; daggers $(\dagger$ and $\dagger \dagger$ ) indicate values significantly different at $P<0.05$ and 0.01 , respectively. and Filloux 2016). Furthermore, a positive correlation has been reported between extra- and intracellular levels of Krebs cycle intermediates such as fumarate, succinate, and 2-oxoglutarate and the regulation of secondary metabolism via the expression of GacA-dependent sRNAs in P. protegens (Takeuchi et al. 2009). However, other signals may exist. This study focused on GABA, which is overproduced in a gacA mutant but is under-represented in a retS mutant of strain CHA0 (Takeuchi et al. 2012).

Using simple in-vitro culture systems, the function of the $g a b T$ gene was found to be instrumental for intracellular GABA levels in $P$. protegens, i.e., the gabT gene encoded GABAT, which catabolized GABA (Table 1). High GABA levels prolonged planktonic growth and diminished biofilm formation (Fig. 3). A root colonization assay under gnotobiotic conditions revealed that accumulated intracellular GABA promoted root colonization by strain CHA0 (Table 2). This result may be rationalized by assuming that GABA delays the action of the Gac/Rsm system during the biofilm phase by negatively affecting the GacS/GacA two component system; however, the underlying mechanisms remain unclear. With a delay in Gac/Rsm activity, i.e., under conditions in which the costly production of secondary metabolites and biofilm formation are attenuated, cells may engage more extensively in proliferation and planktonic modes of growth, which is preferable for root colonization. Previous studies reported that the $\mathrm{gacS} / \mathrm{gacA}$ mutants of P. fluorescens F113 and P. brassicacearum exhibited better root colonization abilities than the wild type under gnotobiotic conditions (Achouak et al. 2004; Lalaouna et al. 2012; Sánchez-Contreras et al. 2002).

GABA concentrations in the rhizosphere in natural soil are an important factor that needs to be considered from a biocontrol point of view. GABA is naturally present in the rhizosphere, and, although few studies have examined its concentration, a low micromolar level is plausible (Moe 2013). In the present study, GABA was used at concentrations of 2 and $5 \mathrm{mM}$ to investigate its function as an intracellular signal; however, the potential of GABA to function as an extracellular signal cannot be excluded. The identification of GABA sensors or receptors that may respond to exogenous GABA at lower concentrations will be a challenge for future studies.

In nutrient-rich, liquid, or semisolid laboratory media and on gnotobiotic plant roots, $P$. protegens, $P$. brassicacearum, $P$. fluorescens F113, and related strains showed signs of unusual genetic instability, with the frequent selection of spontaneous gacS and gacA mutants (Achouak et al. 2004; Bull et al. 2001; Lalaouna et al. 2012; Sánchez-Contreras et al. 2002; Song et al. 2016; Whistler et al. 1998). Although driving selection is not yet fully understood, the gacS and gacA mutants may benefit from an extended phase of cell mass proliferation. In the defined medium used, the gacA mutant did not grow more extensively

Table 2. Effects of a gabT mutation on the ability of Pseudomonas protegens $\mathrm{CHA} 0$ to colonize cucumber roots

\begin{tabular}{lcccc}
\hline & \multicolumn{4}{c}{$\begin{array}{c}\text { Colonization by } \boldsymbol{P} \text {. protegens } \\
\left(\log _{10} \text { CFU per gram of root }\right)\end{array}$} \\
\cline { 2 - 5 } $\begin{array}{l}\text { Bacterial } \\
\text { strain added }\end{array}$ & $\mathbf{6}$ days & $\mathbf{8}$ days & $\mathbf{1 0}$ days & 12 days \\
\hline $\begin{array}{c}\text { CHA0 } \\
\text { (wild type) }\end{array}$ & $7.16 \pm 0.11$ & $6.99 \pm 0.06$ & $7.70 \pm 0.08$ & $7.22 \pm 0.10$ \\
$\Delta g a b T$ & $7.05 \pm 0.12$ & $7.32 \pm 0.02^{*}$ & $7.86 \pm 0.14^{*}$ & $7.46 \pm 0.19^{*}$ \\
\hline
\end{tabular}

${ }^{\text {a }} P$. protegens strains were added at $1 \times 10^{3} \mathrm{CFU}$ per seedling in $50-\mathrm{ml}$ Falcon tubes, each containing $20 \mathrm{ml}$ of one-fifth strength Murashige and Skoog medium with $0.6 \%$ agar and $0.05 \%$ morpholineethanesulfonic acid buffer ( $\mathrm{pH}$ 6.0), after planting two 48-h-old, sterile-grown cucumber seedlings per tube. Plants were harvested after the days indicated. Asterisks (*) denote values that are significantly different from that in $\mathrm{CHA} 0$ at the $P<0.05$ level ( $t$ test).

b Data represent the averages of four to six replicates per treatment, and, thus, a total of eight to 12 plants. 
but had a markedly shorter lag phase than the wild type. Under the in-vitro conditions, gacA mutants (temporarily) dominated by optimizing the planktonic growth phase. In contrast, under natural soil conditions, in which this selection has not been observed, the developmental process leading to biofilm formation will dominate.

In $A$. tumefaciens, the quorum-sensing signal $N$-(3-oxooctanoyl)-homoserine lactone positively regulates virulence, i.e., plant tumor formation. GABA induces AttM lactonase, which degrades $\mathrm{N}$-acyl-homoserine lactones. Thus, GABA — of plant or bacterial origin-quenches quorum sensing and, thus, modulates virulence (Chevrot et al. 2006). P. protegens and several other fluorescent pseudomonads (but not $P$. aeruginosa) lack $N$-acylhomoserine lactone-dependent quorum-sensing machinery; however, at high cell population densities, the Gac/Rsm system provides a quorum sensing-like response (Haas and Keel 2003). It currently remains unclear whether GABA acts on some component of the Gac/Rsm cascade in strain CHA0. In contrast, as shown in the present study, the Gac/Rsm system affects the accumulation of GABA in a similar manner to its effects on secondary metabolism and the production of extracellular macromolecules.

\section{MATERIALS AND METHODS}

\section{Bacterial strains and growth conditions.}

The bacterial strains and plasmids used are listed in Supplementary Table S3. Strains of E. coli and P. protegens were routinely grown in nutrient yeast broth (NYB) $(2.5 \%$ [wt/vol] nutrient broth, $0.5 \%$ [wt/vol] yeast extract) and Luria-Bertani medium with shaking or on nutrient agar plates $(4 \%$ [wt/vol] blood agar base, $0.5 \%$ [wt/vol] yeast extract) amended with the following antibiotics, when required: ampicillin, $100 \mu \mathrm{g} / \mathrm{ml}$ (only for $E$. coli); kanamycin, $25 \mu \mathrm{g} / \mathrm{ml}$; or tetracycline, $25 \mu \mathrm{g} / \mathrm{ml}(125 \mu \mathrm{g} / \mathrm{ml}$ for selection of $P$. protegens). To evaluate the GABA utilization abilities of the strains tested, we used modified M9 minimal medium agar plates (per liter: $\mathrm{Na}_{2} \mathrm{HPO}_{4}, 6.0 \mathrm{~g} ; \mathrm{KH}_{2} \mathrm{PO}_{4}, 3.0 \mathrm{~g}$; $\mathrm{NaCl}, 0.5 \mathrm{~g}$; $\mathrm{MgSO}_{4} \cdot 7 \mathrm{H}_{2} \mathrm{O}, 0.494 \mathrm{~g}$, and $\mathrm{CaCl}_{2} \cdot 2 \mathrm{H}_{2} \mathrm{O}, 0.015 \mathrm{~g}$ ) supplemented with $20 \mathrm{mM}$ GABA as the sole carbon and nitrogen source. In other assays, bacteria were grown in MG medium containing (per liter): mannitol, $10 \mathrm{~g}$; L-glutamic acid, $2.0 \mathrm{~g} ; \mathrm{KH}_{2} \mathrm{PO}_{4}, 0.5 \mathrm{~g} ; \mathrm{NaCl}$, $0.2 \mathrm{~g} ; \mathrm{MgSO}_{4} \cdot 7 \mathrm{H}_{2} \mathrm{O}, 0.494 \mathrm{~g}$, final pH 7 (Park et al. 2010). Incubation temperatures were $30^{\circ} \mathrm{C}$ for $P$. protegens and $37^{\circ} \mathrm{C}$ for E. coli. P. protegens was grown at $35^{\circ} \mathrm{C}$ to improve its capacity to accept heterologous DNA (e.g., in electro-transformation or in mating with E. coli).

\section{Construction of gabD'-'lacZ- and gabDT'-'lacZ- fusions.}

A 420-bp EcoRI-BamHI fragment from the upstream region of PFLCHA0_c01870 ( $g a b D)$, including the first five codons of the $g a b D$ gene and presumably containing the $g a b D$ promoter, was amplified using the primers GabDProFEcoRI and GabDProR (Supplementary Table S4), and the amplified fragment was cloned into pCR-Blunt II-TOPO (Invitrogen). The insert obtained was confirmed by sequencing and was digested with $E c o$ RI and BamHI. This fragment was fused in-frame to the eighth codon of the lacZ gene in vector pME6014 to obtain pME6014gabD, containing a translational gabD'- 'lacZ fusion (Fig. 1A).

A $2.0-\mathrm{kb}$ BamHI-Pst I fragment containing the region from $0.4 \mathrm{~kb}$ upstream of PFLCHA0_c01870 ( $\mathrm{gabD})$ to the $5^{\prime}$ region of PFLCHA0_c01880 ( $g a b T)$ including the first six codons of the gabT gene was amplified, using the primers GabDProFBamHI and GabTProR, and was inserted into pBluescript II KS (Stratagene). The insert obtained was confirmed by sequencing, and, for the mutational introduction of a conventional Shine-Dalgarno sequence (i.e., the modification of TAAGGAAG to AGGA), this construct was used as a template plasmid for polymerase chain reaction (PCR) with the mutagenic primers GabDggaF and GabDggaR designed for the amplification of a circular product. The mutation was confirmed by sequencing. The native and mutated regions were fused to the eighth codon of the lac $Z$ gene in the vector pME6014 (at the BamHI and PstI sites) to obtain pME6014gabDT and pME6014gabDTgga, respectively, containing a translational gabDT' - 'lacZ fusion (Fig. 1B).

\section{Generation of $g a b D$ - and gabT-negative mutants.}

In-frame deletions in the chromosomal $g a b D$ and $g a b T$ genes of $P$. protegens CHA0 were created as follows. Fragments of approximately 800 to 850 bp located on each side of the $g a b D$ or gabT gene were amplified by PCR with the primer pairs GabDUF/ GabDUR and GabDDF/GabDDR for $g a b D$ and GabTUF/GabTUR and GabTDF/GabTDR for $g a b T$. Each of the two corresponding fragments were ligated and amplified as 1.6 to $1.7-\mathrm{kb}$ fragments, using the primers GabDUF/GabDDR or GabTUF/GabTDR, respectively. The amplified fragments were cloned into pCR-Blunt II-TOPO. After confirmation by sequencing, these fragments were subcloned into pME3087 cut with BamHI and HindIII, to give pME3087gabD and pME3087gabT. These plasmids were mobilized from $E$. coli $\mathrm{DH} 5 \alpha$ to $P$. protegens $\mathrm{CHA} 0$ by triparental mating with E. coli HB101/pME497 and were selected for tetracycline resistance. Excision of the vector via a second crossingover was performed after enrichment for tetracycline-sensitive cells (Humair et al. 2009), generating the gabD or gabT mutant.

\section{Complementation of the gabT-negative mutant.}

To restore $g a b T$ function in the gabT mutant, a $3.8-\mathrm{kb}$ fragment containing the region from $0.4 \mathrm{~kb}$ upstream of $g a b D$ to $0.5 \mathrm{~kb}$ downstream of gabT was amplified with the primers GabDProFBamHI and GabTDR. The amplified fragment was cloned into pCR-Blunt II-TOPO and the insert obtained was confirmed by sequencing and was digested with BamHI and HindIII. This fragment was subcloned into pME6031 cleaved at the BamHI and HindIII sites. The resultant plasmid, pME6031gabT, was introduced into the gabT mutant by electroporation.

\section{$\beta$-Galactosidase assays.}

$\beta$-Galactosidase activities were quantified by the Miller method (Miller 1972). $P$. protegens strains were grown at $30^{\circ} \mathrm{C}$ in $50-\mathrm{ml}$ flasks containing $20 \mathrm{ml}$ of NYB amended with $0.05 \%$ Triton $\mathrm{X}-100$, with shaking, at $180 \mathrm{rpm}$. Triton $\mathrm{X}-100$ was required to prevent cell aggregation.

\section{GABA extraction and quantification by HPLC.}

Intracellular levels of GABA were assessed in $P$. protegens strains cultured in MG medium supplemented with GABA at a concentration of $5 \mathrm{mM}$ (MG + GABA). For the preculture, the strains were grown overnight in NYB medium and were rinsed twice with MG medium without GABA. The strains were then inoculated at a starting OD of 0.3 in $10 \mathrm{ml}$ fresh $\mathrm{MG}+\mathrm{GABA}$ medium in $50-\mathrm{ml}$ flasks and were grown at $180 \mathrm{rpm}$ and $30^{\circ} \mathrm{C}$ for $15 \mathrm{~h}$. In each strain, three cultures were incubated in parallel. Cells were harvested by centrifugation at $5,000 \times g$ for $10 \mathrm{~min}$, and the pellet was washed twice with MG medium without GABA and was preserved at $-80^{\circ} \mathrm{C}$ for GABA extraction. The pellets were homogenized in $750 \mu \mathrm{l}$ of $70 \%$ methanol and centrifuged at $10,000 \times g$ for $10 \mathrm{~min}$ at $4^{\circ} \mathrm{C}$. The supernatant was dried and preserved at $-80^{\circ} \mathrm{C}$.

Regarding the detection of GABA, the metabolite extract was labeled with 4-dimethyl-aminoazobenzene-4'-sulfonyl (dabsyl) chloride (Thermo Fisher Scientific K.K.), following the method described by Abe et al. (1998). The metabolite extract was dissolved in $200 \mu \mathrm{l}$ of $0.1 \mathrm{M}$ sodium hydrogen carbonate buffer (pH 9.0), and $200 \mu \mathrm{l}$ of $2 \mathrm{mM}$ dabsyl chloride solution in acetone was added. The dabsylation reaction was performed at 
$70^{\circ} \mathrm{C}$ for $20 \mathrm{~min}$. After vacuum evaporation, the dried samples were dissolved in $100 \mu \mathrm{l}$ of $100 \%$ ethanol. The concentration of GABA was assessed by HPLC on a Waters system (Waters) consisting of a photodiode array detector 996 and pump 616, equipped with a normal phase column (TSKgel ODS-80Ts column, $4.6 \times 250 \mathrm{~mm}$, Tosoh). Routine chromatography was performed at $28^{\circ} \mathrm{C}$, using a solvent system consisting of $50 \mathrm{mM}$ sodium acetate ( $\mathrm{pH} 4.15)$ (solvent $\mathrm{A}$ ) and acetonitrile (solvent B). Elution was performed with the concentration of solvent B as follows: 0 to $40 \mathrm{~min}$, linear gradient from 35 to $40 \%$; 40 to $80 \mathrm{~min}$, isocratic at $40 \%$. The column was then washed with a solution of 5\% solvent A and $95 \%$ solvent B for $20 \mathrm{~min}$ and was reconditioned with a solution of $75 \%$ solvent $A$ and $35 \%$ solvent B. Dabsyl derivatives of amino acids were detected at $430 \mathrm{~nm}$. The concentration of GABA was expressed relative to cell density $\left(\mathrm{OD}_{600 \mathrm{~nm}}\right.$ is approximately $\left.1.0 / \mathrm{ml}\right)$. Regarding the standard, GABA was obtained from Wako and was labeled with dabsyl chloride in the same manner described above.

\section{Enzyme assays.}

To prepare cell extracts, cultures of $P$. protegens $\mathrm{CHA} 0$ or the mutant strains were grown on MG medium supplemented with GABA at $5 \mathrm{mM}$, under the same conditions as described above. Cells were harvested at $4^{\circ} \mathrm{C}$ by centrifugation at $5,000 \times g$ for $10 \mathrm{~min}$ and the pellet was suspended in $5 \mathrm{ml}$ of $20 \mathrm{mM}$ phosphate buffer ( $\mathrm{pH}$ 7.2) and was centrifuged again. Cells were then resuspended in $1 \mathrm{ml}$ of breaking buffer (40 mM HEPES, $1 \mathrm{mM}$ dithiothreitol [DTT] at $\mathrm{pH} \mathrm{7.0)}$ and were sonicated on ice for $1 \mathrm{~min}$. Cell debris was removed by centrifugation at $10,000 \times g$ for $10 \mathrm{~min}$. Protein concentrations were assessed by the Bradford method.

2-Oxoglutarate-dependent GABAT activity was measured with $150 \mathrm{mM}$ GABA and $50 \mathrm{mM}$ 2-oxoglutarate as substrates, as described earlier by Bartsch et al. (1990) and Prell et al. (2002). The assay was performed in a final volume of $300 \mu \mathrm{l}$, using $150 \mu \mathrm{l}$ of the cell extract. Glutamate formation was assessed by the reduction of $\mathrm{NAD}^{+}$in a coupled glutamate dehydrogenase (GDH) assay. In a final volume of $450 \mu \mathrm{l}(40 \mathrm{mM}$ hydrazine, $50 \mathrm{mM}$ glycine [pH 7.6], $2.7 \mathrm{mM} \mathrm{NAD}^{+}, 1$ unit GDH), the complete GABAT sample $(150 \mu \mathrm{l})$ was incubated at $30^{\circ} \mathrm{C}$ for $2 \mathrm{~h}$. NADH production was measured at $340 \mathrm{~nm}$ in a spectrophotometer. Assays were performed with a control lacking GDH for each extract. Results were compared with a standard curve (with 0-1 $\mu \mathrm{mol}$ glutamate per sample).

SSDH was measured with $2.5 \mathrm{mM}$ succinate semialdehyde and $1 \mathrm{mM} \mathrm{NAD}^{+}$as substrates. The assay was performed in $50 \mathrm{mM}$ Tris- $\mathrm{HCl}$ buffer at $\mathrm{pH} 8.0$ and $1 \mathrm{mM}$ DTT. The reduction of NAD ${ }^{+}$ was measured spectrophotometrically at $340 \mathrm{~nm}$. Assays were performed with a control lacking succinate semialdehyde for each extract.

\section{Biofilm assay.}

Cells from overnight cultures grown in NYB were washed once in saline $(0.9 \% \mathrm{NaCl})$ and were resuspended to an $\mathrm{OD}_{600}$ of 3.0 in saline. One-microliter aliquots were inoculated into 96-well polypropylene plates filled with MG medium $(200 \mu \mathrm{l}$ per well) without GABA or supplemented with GABA at 2 or $5 \mathrm{mM}$. Cultures were incubated statically at room temperature, with a cover, in a container with a water-soaked paper towel to prevent evaporation. After $24 \mathrm{~h}, 100 \mu \mathrm{l}$ of the planktonic cell cultures was used to measure $\mathrm{OD}_{600}$ values. Fifty microliters of a $0.5 \%$ solution of crystal violet was added to each well to stain the biofilm, the plates were incubated at room temperature for $15 \mathrm{~min}$, and were then rinsed thoroughly and repeatedly with water. Regarding quantification, the stained biofilm of each well was suspended in $400 \mu \mathrm{l}$ of $95 \%$ ethanol, followed by the addition of $600 \mu \mathrm{l}$ of water, and absorbance was measured at $600 \mathrm{~nm}$

\section{Swarming motility assay.}

Cells from overnight cultures grown in NYB were washed once in saline $(0.9 \% \mathrm{NaCl})$ and were resuspended to an $\mathrm{OD}_{600}$ of 3.0 in saline. A sterile toothpick was then dipped into the cell suspension and was used to inoculate by stabbing vertically into the center of semisolid MG agar $(0.5 \%)$ plates containing 0,2 , or $5 \mathrm{mM}$ GABA. The plates were incubated at room temperature for $60 \mathrm{~h}$, and swarming motility was assessed by examining the circular lawn formed by the motile bacterial cells.

\section{Plant disease suppression and root-colonization assays.}

Flasks containing $20 \mathrm{~g}$ of nonsterile soil (vermiculite) were each planted with three seedlings of cucumber (Cucumis sativus L. cv. Tokiwajibai) and were treated with one or both Pythium ultimum MAFF425494 and $P$. protegens. $P$. protegens strains were added to soil as a suspension ( $4 \mathrm{ml}$ per flask) of cells washed twice in sterile distilled water, to give $2 \times 10^{7} \mathrm{CFU}$ per gram of soil. Control flasks received the same amount of sterile water. Seedlings were covered with $5 \mathrm{~g}$ of nontreated soil and flasks were sealed with aerated silicon caps. The microcosms were incubated in a growth chamber containing $60 \%$ relative humidity at $26^{\circ} \mathrm{C}$ with light for $16 \mathrm{~h}$, followed by an 8-h dark period. No watering was necessary. After a 7day incubation, the biocontrol activity of each strain was assessed by counting the number of surviving plants and measuring shoot and root fresh weights per flask. The root colonization of each strain was also simultaneously assessed; whole roots in each flask were rinsed thoroughly and repeatedly with sterile, ultrapure water and were collected in 50-ml conical tubes containing $15 \mathrm{ml}$ of saline. After vigorous agitation, bacteria were enumerated by plating dilutions. Data of both experiments were first analyzed for trial-bytreatment interactions by an analysis of variance, which indicated that the data from the two independent trials could be pooled. Means were separated using Tukey's highly significant difference test (at $P \leq 0.05$ ). Statistical analyses were performed using $\mathrm{R}$ (version 3.0.1).

\section{Gnotobiotic plant root-colonization assay.}

Two 48-h-old, sterile-grown cucumber seedlings were planted in $20 \mathrm{ml}$ of fifth-strength Murashige and Skoog agar medium (0.6\%) (Murashige and Skoog 1962) supplemented with 0.05\% morpholineethanesulfonic acid buffer $(\mathrm{pH}$ 6.0) in 50-ml conical tubes (Becton Dickinson/Falcon). Bacterial cells from overnight cultures grown in NYB were washed once in sterile, ultrapure water and were adjusted to a final concentration of approximately $1.0 \times 10^{4}$ $\mathrm{CFU} / \mathrm{ml}$. Two hundred-microliter aliquots were inoculated into seedlings to give $1.0 \times 10^{3} \mathrm{CFU}$ per seedling, and the tubes were loosely capped. As a control, six plants were treated with $200 \mu \mathrm{l}$ of sterilized water.

Seedlings were incubated in a growth chamber at $26^{\circ} \mathrm{C}$ with light for $16 \mathrm{~h}$, followed by an 8-h dark period. After 6, 8, 10, and 12-day incubations, whole roots were rinsed thoroughly and repeatedly with sterile, ultrapure water and were collected in 50-ml conical tubes containing $15 \mathrm{ml}$ of saline. After vigorous agitation, bacteria were enumerated by plating dilutions. In water-control plants, no microorganism was confirmed to be colonized on the roots after a 12-day inoculation.

\section{ACKNOWLEDGMENTS}

This study is dedicated to the late Dieter Haas from Universite de Lausanne, who passed away in April 2017, for his careful reading of the manuscript and helpful comments. I thank K. Yamada for help with HPLC assays, S. Heeb for providing bacterial strains, and N. Noda and N. Hattori for their technical assistance. This work was supported in part by the Program for the Promotion 
of Basic Research Activities for Innovative Biosciences (PROBRAIN), by the Science and Technology Research Promotion Program for Agriculture, Forestry, Fisheries and Food Industry, and by the Japan Society for the Promotion of Science KAKENHI grant 15K07318.

\section{LITERATURE CITED}

Abe, T., Kurozumi, Y., Yao, W. B., and Ubuka, T. 1998. High-performance liquid chromatographic determination of $\beta$-alanine, $\beta$-aminoisobutyric acid and $\gamma$-aminobutyric acid in tissue extracts and urine of normal and (aminooxy)acetate-treated rats. J. Chromatogr. B Biomed. Sci. Appl. 712:43-49.

Achouak, W., Conrod, S., Cohen, V., and Heulin, T. 2004. Phenotypic variation of Pseudomonas brassicacearum as a plant root-colonization strategy. Mol. Plant-Microbe Interact. 17:872-879.

Badri, D. V., Chaparro, J. M., Zhang, R., Shen, Q., and Vivanco, J. M. 2013. Application of natural blends of phytochemicals derived from the root exudates of Arabidopsis to the soil reveal that phenolic-related compounds predominantly modulate the soil microbiome. J. Biol. Chem. 288:4502-4512.

Barahona, E., Navazo, A., Martínez-Granero, F., Zea-Bonilla, T., PérezJiménez, R. M., Martín, M., and Rivilla, R. 2011. Pseudomonas fluorescens F113 mutant with enhanced competitive colonization ability and improved biocontrol activity against fungal root pathogens. Appl. Environ. Microbiol. 77:5412-5419.

Bartsch, K., von Johnn-Marteville, A., and Schulz, A. 1990. Molecular analysis of two genes of the Escherichia coli gab cluster: Nucleotide sequence of the glutamate:succinic semialdehyde transaminase gene ( $g a b T)$ and characterization of the succinic semialdehyde dehydrogenase gene (gabD). J. Bacteriol. 172:7035-7042.

Blumer, C., and Haas, D. 2000. Iron regulation of the hcnABC genes encoding hydrogen cyanide synthase depends on the anaerobic regulator ANR rather than on the global activator GacA in Pseudomonas fluorescens CHA0. Microbiology 146:2417-2424.

Bull, C. T., Duffy, B., Voisard, C., Défago, G., Keel, C., and Haas, D. 2001. Characterization of spontaneous gacS and gacA regulatory mutants of Pseudomonas fluorescens biocontrol strain CHAO. Antonie van Leeuwenhoek 79:327-336.

Chevrot, R., Rosen, R., Haudecoeur, E., Cirou, A., Shelp, B. J., Ron, E., and Faure, D. 2006. GABA controls the level of quorum-sensing signal in Agrobacterium tumefaciens. Proc. Natl. Acad. Sci. U.S.A. 103:7460-7464.

Chou, H. T., Kwon, D. H., Hegazy, M., and Lu, C. D. 2008. Transcriptome analysis of agmatine and putrescine catabolism in Pseudomonas aeruginosa PAO1. J. Bacteriol. 190:1966-1975.

Dasu, V. V., Nakada, Y., Ohnishi-Kameyama, M., Kimura, K., and Itoh, Y. 2006. Characterization and a role of Pseudomonas aeruginosa spermidine dehydrogenase in polyamine catabolism. Microbiology 152:2265-2272.

Goodman, A. L., Merighi, M., Hyodo, M., Ventre, I., Filloux, A., and Lory, S. 2009. Direct interaction between sensor kinase proteins mediates acute and chronic disease phenotypes in a bacterial pathogen. Genes Dev. 23:249-259

Haas, D., and Défago, G. 2005. Biological control of soil-borne pathogens by fluorescent pseudomonads. Nat. Rev. Microbiol. 3:307-319.

Haas, D., and Keel, C. 2003. Regulation of antibiotic production in rootcolonizing Peudomonas spp. and relevance for biological control of plant disease. Annu. Rev. Phytopathol. 41:117-153.

Heeb, S., Blumer, C., and Haas, D. 2002. Regulatory RNA as mediator in GacA/RsmA-dependent global control of exoproduct formation in Pseudomonas fluorescens CHA0. J. Bacteriol. 184:1046-1056.

Humair, B., González, N., Mossialos, D., Reimmann, C., and Haas, D. 2009. Temperature-responsive sensing regulates biocontrol factor expression in Pseudomonas fluorescens CHA0. ISME J. 3:955-965.

Kay, E., Dubuis, C., and Haas, D. 2005. Three small RNAs jointly ensure secondary metabolism and biocontrol in Pseudomonas fluorescens CHA0. Proc. Natl. Acad. Sci. U.S.A. 102:17136-17141.

Kay, E., Humair, B., Dénervaud, V., Riedel, K., Spahr, S., Eberl, L., Valverde, C., and Haas, D. 2006. Two GacA-dependent small RNAs modulate the quorum-sensing response in Pseudomonas aeruginosa. J. Bacteriol. 188:6026-6033

Kidarsa, T. A., Shaffer, B. T., Goebel, N. C., Roberts, D. P., Buyer, J. S., Johnson, A., Kobayashi, D. Y., Zabriskie, T. M., Paulsen, I., and Loper, J. E. 2013. Genes expressed by the biological control bacterium Pseudomonas protegens Pf-5 on seed surfaces under the control of the global regulators GacA and RpoS. Environ. Microbiol. 15:716-735.

Kim, Y. C., Leveau, J., McSpadden Gardener, B. B., Pierson, E. A., Pierson, L. S., 3rd, and Ryu, C. M. 2011. The multifactorial basis for plant health promotion by plant-associated bacteria. Appl. Environ. Microbiol. 77: 1548-1555.
Kulasakara, H., Lee, V., Brencic, A., Liberati, N., Urbach, J., Miyata, S., Lee, D. G., Neely, A. N., Hyodo, M., Hayakawa, Y., Ausubel, F. M., and Lory, S. 2006. Analysis of Pseudomonas aeruginosa diguanylate cyclases and phosphodiesterases reveals a role for bis- $\left(3^{\prime}-5^{\prime}\right)$-cyclicGMP in virulence. Proc. Natl. Acad. Sci. U.S.A. 103:2839-2844.

Kupferschmied, P., Maurhofer, M., and Keel, C. 2013. Promise for plant pest control: Root-associated pseudomonads with insecticidal activities. Front. Plant Sci. 4:287.

Lalaouna, D., Fochesato, S., Sanchez, L., Schmitt-Kopplin, P., Haas, D., Heulin, T., and Achouak, W. 2012. Phenotypic switching in Pseudomonas brassicacearum involves GacS- and GacA-dependent Rsm small RNAs. Appl. Environ. Microbiol. 78:1658-1665.

Lapouge, K., Schubert, M., Allain, F. H.-T., and Haas, D. 2008. Gac/Rsm signal transduction pathway of $\gamma$-proteobacteria: From RNA recognition to regulation of social behaviour. Mol. Microbiol. 67:241-253.

Lapouge, K., Sineva, E., Lindell, M., Starke, K., Baker, C. S., Babitzke, P., and Haas, D. 2007. Mechanism of henA mRNA recognition in the Gac/Rsm signal transduction pathway of Pseudomonas fluorescens. Mol. Microbiol. 66:341-356.

Laville, J., Voisard, C., Keel, C., Maurhofer, M., Défago, G., and Haas, D. 1992. Global control in Pseudomonas fluorescens mediating antibiotic synthesis and suppression of black root rot of tobacco. Proc. Natl. Acad. Sci. U.S.A. 89:1562-1566.

Ma, J., Campbell, A., and Karlin, S. 2002. Correlations between ShineDalgarno sequences and gene features such as predicted expression levels and operon structures. J. Bacteriol. 184:5733-5745.

Miller, J. H. 1972. Experiments in Molecular Genetics. Cold Spring Harbor Laboratory Press, Cold Spring Harbor, NY, U.S.A.

Moe, L. A. 2013. Amino acids in the rhizosphere: From plants to microbes. Am. J. Bot. 100:1692-1705.

Murashige, T., and Skoog, F. 1962. A revised medium for the rapid growth and bioassay with tobacco tissue cultures. Physiol. Plant. 15: 473-497.

Park, D. H., Mirabella, R., Bronstein, P. A., Preston, G. M., Haring, M. A., Lim, C. K., Collmer, A., and Schuurink, R. C. 2010. Mutations in $\gamma$-aminobutyric acid (GABA) transaminase genes in plants or Pseudomonas syringae reduce bacterial virulence. Plant J. 64:318-330.

Parkins, M. D., Ceri, H., and Storey, D. G. 2001. Pseudomonas aeruginosa $\mathrm{GacA}$, a factor in multihost virulence, is also essential for biofilm formation. Mol. Microbiol. 40:1215-1226.

Prell, J., Boesten, B., Poole, P., and Priefer, U. B. 2002. The Rhizobium leguminosarum bv. viciae VF39 $\gamma$-aminobutyrate (GABA) aminotransferase gene $(g a b T)$ is induced by GABA and highly expressed in bacteroids. Microbiology 148:615-623.

Reimmann, C., Valverde, C., Kay, E., and Haas, D. 2005. Posttranscriptional repression of $\mathrm{GacS} / \mathrm{GacA}$-controlled genes by the RNA-binding protein RsmE acting together with RsmA in the biocontrol strain Pseudomonas fluorescens CHA0. J. Bacteriol. 187:276-285.

Sánchez-Contreras, M., Martín, M., Villacieros, M., O’Gara, F., Bonilla, I., and Rivilla, R. 2002. Phenotypic selection and phase variation occur during alfalfa root colonization by Pseudomonas fluorescens F113. J. Bacteriol. 184:1587-1596.

Schneider, B. L., Ruback, S., Kiupakis, A. K., Kasbarian, H., Pybus, C., and Reitzer, L. 2002. The Escherichia coli gabDTPC operon: Specific $\gamma$-aminobutyrate catabolism and nonspecific induction. J. Bacteriol. 184: 6976-6986.

Song, C., Kidarsa, T. A., van de Mortel, J. E., Loper, J. E., and Raaijmakers, J. M. 2016. Living on the edge: Emergence of spontaneous gac mutations in Pseudomonas protegens during swarming motility. Environ. Microbiol. 18:3453-3465.

Takeuchi, K., Kiefer, P., Reimmann, C., Keel, C., Dubuis, C., Rolli, J., Vorholt, J. A., and Haas, D. 2009. Small RNA-dependent expression of secondary metabolism is controlled by Krebs cycle function in Pseudomonas fluorescens. J. Biol. Chem. 284:34976-34985.

Takeuchi, K., Yamada, K., and Haas, D. 2012. ppGpp controlled by the Gac/Rsm regulatory pathway sustains biocontrol activity in Pseudomonas fluorescens CHA0. Mol. Plant-Microbe Interact. 25:1440-1449.

Valentini, M., and Filloux, A. 2016. Biofilms and cyclic di-GMP (c-diGMP) signaling: Lessons from Pseudomonas aeruginosa and other bacteria. J. Biol. Chem. 291:12547-12555.

Valverde, C., Heeb, S., Keel, C., and Haas, D. 2003. RsmY, a small regulatory RNA, is required in concert with RsmZ for GacA-dependent expression of biocontrol traits in Pseudomonas fluorescens CHA0. Mol. Microbiol. 50:1361-1379.

Whistler, C. A., Corbell, N. A., Sarniguet, A., Ream, W., and Loper, J. E. 1998. The two-component regulators GacS and GacA influence accumulation of the stationary-phase sigma factor $\sigma^{\mathrm{S}}$ and the stress response in Pseudomonas fluorescens Pf-5. J. Bacteriol. 180:6635-6641. 
Winsor, G. L., Lam, D. K., Fleming, L., Lo, R., Whiteside, M. D., Yu, N. Y., Hancock, R. E., and Brinkman, F. S. 2011. Pseudomonas Genome database: Improved comparative analysis and population genomics capability for Pseudomonas genomes. Nucleic Acids Res. 39:D596-D600.

Workentine, M. L., Chang, L., Ceri, H., and Turner, R. J. 2009. The GacS-GacA two-component regulatory system of Pseudomonas fluorescens: A bacterial two-hybrid analysis. FEMS Microbiol. Lett. 292:50-56
Yao, X., He, W., and Lu, C. D. 2011. Functional characterization of seven $\gamma$-glutamylpolyamine synthetase genes and the bauRABCD locus for polyamine and $\beta$-alanine utilization in Pseudomonas aeruginosa PAO1. J. Bacteriol. 193:3923-3930.

\section{AUTHOR-RECOMMENDED INTERNET RESOURCE}

Pseudomonas Genome database: http://www.pseudomonas.com 